\title{
PCR DETECTION OF STRUCTURALLY ABNORMAL Y CHROMOSOMES
}

\author{
Shigeo NAGAFUchI, ${ }^{1}$ Satoko SeKI, ${ }^{1}$ Yutaka NAKAHORI, ${ }^{2}$ \\ Takashi Tamura, ${ }^{1,3}$ Hironao Numabe, ${ }^{1,4}$ and Yasuo NaKagome ${ }^{2}$,* \\ 'Department of Congenital Abnormalities Research, National Children's \\ Medical Research Center, Taishido, Setagaya-ku, Tokyo 154, Japan \\ ${ }^{2}$ Department of Human Genetics, School of International Health, \\ University of Tokyo, Hongo, Bunkyo-ku, Tokyo 113, Japan \\ ${ }^{3}$ Department of Clinical Genetics, School of Health Sciences, \\ Kyorin University, Miyashita-cho, Hachioji, Tokyo 192, Japan \\ ${ }^{4}$ Department of Pediatrics, Tokyo Medical College, \\ Shinjuku, Shinjuku-ku, Tokyo 160, Japan
}

\begin{abstract}
Summary Three probes each detecting a locus on the proximal long arm of the $\mathrm{Y}$ chromosome were partially sequenced. Thus, 3 sets of novel primers were developed which enable PCR detection of these 3 loci. Five previously reported primer sets, 3 on the short arm and each one on the centromere and the distal long arm, were mapped along with the novel three using a mapping panel consisted of 8 patients each with different structural abnormality of the $Y$ chromosome, Now, PCR detection of these 8 loci covering an entire length of the $\mathrm{Y}$ chromosome has become possible enabling rapid screening of patients with $Y$ chromosome aberrations.
\end{abstract}

Key Words PCR, STS, Y chromosome, deletion mapping

\section{INTRODUCTION}

Genetic linkage studies of the non-pseudoautosomal region of the $Y$ chromosome are not possible because it does not form chiasma in meiosis. Therefore, deletion mapping is a necessary tool in the characterization of the human $\mathrm{Y}$ chromosome. Construction of a deletion map by Southern blotting using over ten different probes is laborious. However, the use of polymerase chain reaction (PCR) described by Saiki et al. (1988) should enable the analysis of many samples in a very short period of time.

We have developed 3 novel primer sets which detect 3 loci within the non-

Received June 1, 1992; Accepted June 22, 1992.

*To whom correspondence should be addressed. 
fluorescent part of the long arm of the $\mathrm{Y}$ chromosome. As far as the authors are aware of, PCR detection of only one locus has been possible within this part of the $\mathrm{Y}$ chromosome (Lucotte et al., 1991).

\section{MATERIALS AND METHODS}

Subcloning and sequencing of probes. Three probes were subcloned into the vector PUC18 and partially sequenced. The 3 probes detect 3 loci within a nonfluorescent part of the long arm of the Y chromosome. The probe 87-26 which detects locus DYS139 was digested with $X b a \mathrm{I}$ and a $1.65 \mathrm{~kb}$ fragment was subcloned into the $X b a I$ site of the vector pUC18. The probe 87-19 (DYS132) was digested with HindIII and a $0.42 \mathrm{~kb}$ similarly subcloned in the HindIII site of pUC18. These 2 probes were originally described by Nakahori et al. (1991a) in which we estimated the size of the fragments slightly shorter. A $0.8 \mathrm{~kb}$ fragment was derived from the prove $49 \mathrm{f}$ (DYS1) by digestion with both EcoRI and XbaI. The fragment was subcloned into the pUC18 vector digested with these 2 enzymes. The 49 f probe was originally described by Vergnaud et al. (1986) and a gift from Dr. J. Weissenbach.

Using a set of a universal and a reverse primer (Toyobo, Tokyo) spanning cloning sites of the vector pUC18, each of the 3 insert was partially sequenced by the dideoxy chain-termination method using the Sequenase Sequencing Kit (Toyobo). Out of about $170 \mathrm{bp}$ sequences each representing either end of individual inserts, a $20-23$ bp primer sequence was chosen. A set of two primers representing near both ends of an insert was designed to match in their length and as much as possible in their GC contents.

Using normal male and female DNA, PCR reactions were carried out using the 3 primer sets. The annealing temperature of each PCR reaction was set at $50^{\circ} \mathrm{C}$ and when a single male (Y)-specific band was not observed, a higher temperature was applied. When the use of a $65^{\circ} \mathrm{C}$-annealing temperature was not successful in obtaining a distinct $Y$-specific band, different primer sequences were attempted.

Additional 5 loci were also examined by PCR reaction. Three loci were on the short arm, PABY, SRY, and AMGL. As to the PABY locus, a mixture of the PABA, PABB, and PABC primers simultaneously detects both $X$-specific (PABX) and Y-specific (PABY) sequences from the proximal border of the pseudoautosomal region (Ellis et al., 1990). The SRY primers have been described by Nakagome et al. (1991b) and are based on the sequences reported by Sinclair et al. (1990). The AMGL primers were designed from the sequences of the amelogenin gene (Nakahori et al., 1991b), and the X-Y homologous primer sequences have been reported elsewhere (Nakahori et al., 1991c). The DYZ3 locus close to the centromere was examined using $\mathrm{Y} 1$ and $\mathrm{Y} 2$ primers (Witt and Erickson, 1989). A primer set which detect the B segment of the DYZ1 locus was also used 
PCR DETECTION OF Y CHROMOSOMES

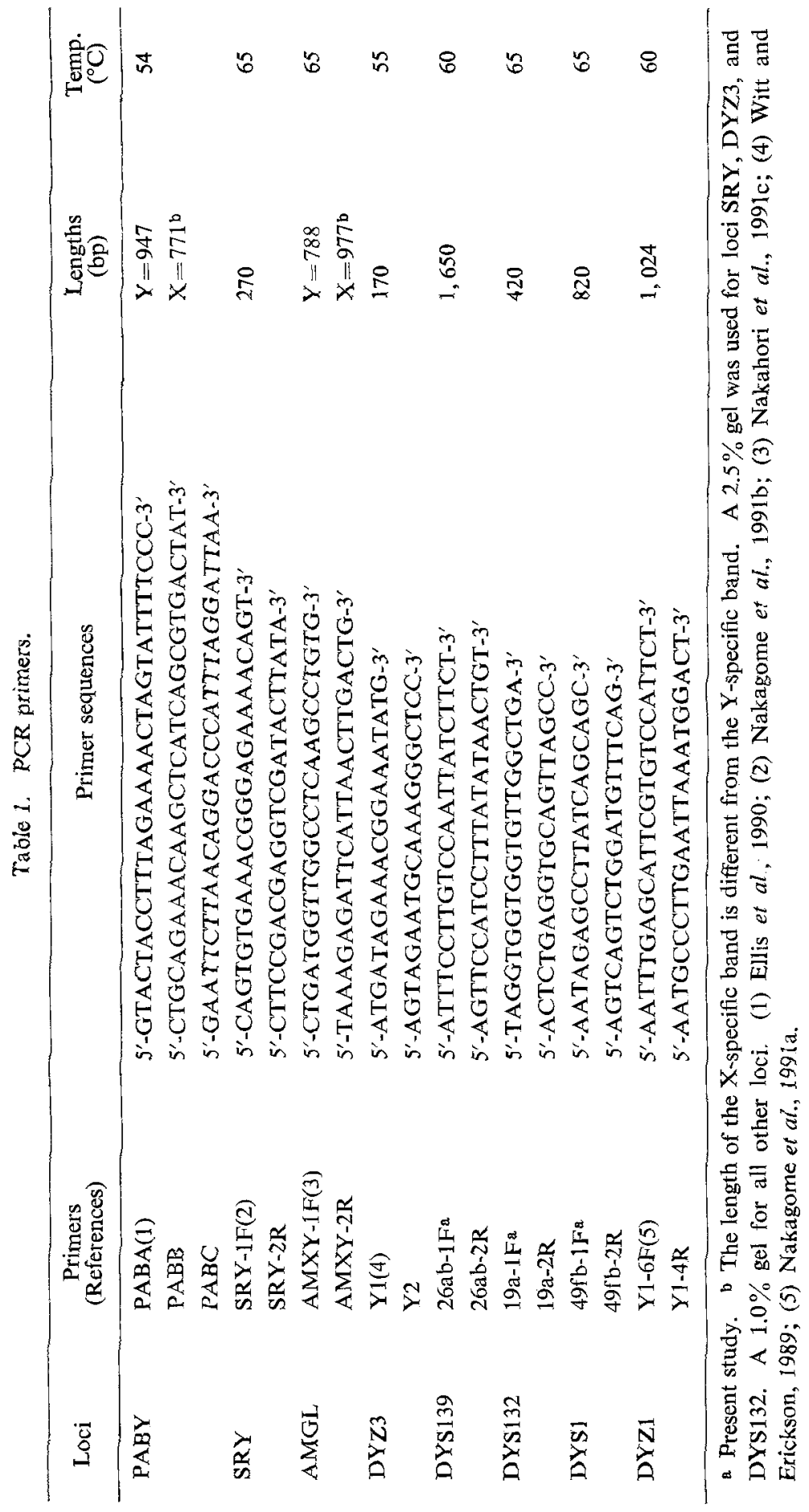

Vol. 37, No. 3, 1992 
(Nakagome et al., 199la). The locus represents a part of the repeating unit of the DYZI family on the distal long arm (Cooke, 1976; Nakahori et al., 1986).

PCR procedures. A $50 \mu \mathrm{l}$ of reaction mixture, containing $0.5 \mu \mathrm{g}$ genomic DNA, $5 \mu$ of $10 X$ PCR buffer $\left(500 \mathrm{mM} \mathrm{KCl}, 100 \mathrm{~mm}\right.$ Tris- $\mathrm{HCl} \mathrm{pH} 8.4,15 \mathrm{~mm} \mathrm{MgCl}_{2}$, $200 \mu \mathrm{g} / \mathrm{ml}$ gelatin), $5 \mu \mathrm{l}$ of $8 \mathrm{~mm}$ dNTPs, $5 \mu \mathrm{l}$ each of $10 \mathrm{~mm}$ primers and $0.5 \mu \mathrm{Taq}$ polymerase, were applied to a thermalcycler PJ-1000 (Perkin Elmer Cetus). The reaction mixture was preheated to $94^{\circ} \mathrm{C}$ for $5 \mathrm{~min}$ before the PCR cycles. The cycles were routinely carried out 25 times, as follows: denaturation at $94^{\circ} \mathrm{C}$ for $1 \mathrm{~min}$; annealing at the specific temperature listed in Table 1 for $2 \mathrm{~min}$ and extension at $72^{\circ} \mathrm{C}$ for $2 \mathrm{~min}$. In the case of PABY, the cycle was carried out 30 times and annealing time was $1 \mathrm{~min}$. Electrophoresis was performed on $1 \%$ or $2.5 \%$ agarose gel according to the size of the amplified sequences (see Table 1).

A mapping panel consisted of eight DNA samples from patients with structurally abnormal $\mathrm{Y}$ chromosomes were subjected to PCR amplification. They were cases $877,1,027,1,023,534,815,1,018$, 981, and 924. The results of Southernblot analysis of these samples was previously reported (Nakahori et al., 1991a). Normal male and female samples were amplified as controls. Genomic DNAs were prepared from peripheral blood leukocytes by the standard methods.

\section{RESULTS}

Three novel primer sets were established for the three loci within the nonfluorescent part of the long arm. Their sequences and annealing temperatures were shown in Table 1. As shown in Fig. 1a to 1c, each primer set, including that for the DYS1 locus, produces single distinct band specific to the male. The DYS1 locus has been known to show multiple bands with polymorphism when examined by the Southern-blot method using the $49 \mathrm{f}$ probe (Human Gene Mapping 11).

The presence or absence of amplified sequences by PCR reactions was in complete agreement with the results of Southern analysis described earlier (Nakahori et al., 1991a). Results of PCR detection of the DYZ3 locus corresponded to the presence or absence of $50 \mathrm{f} 2 \mathrm{D}$ locus in Southern blot analysis. The location and the order of the 8 loci detected by the present study are summarized in Fig. 2.

\section{DISCUSSION}

At least two genes, those coding azoospermy factor and growth control $Y$, are thought to be located on the long arm of the $\mathrm{Y}$ chromosome (Human Gene Mapping 11). The construction of a deletion map of the long arm would contribute to the search for these genes. Since deletions in clinical samples can be detected by PCR as well as by Southern analysis, efficient deletion mapping by PCR is possible at those loci for which sequence data is available. 

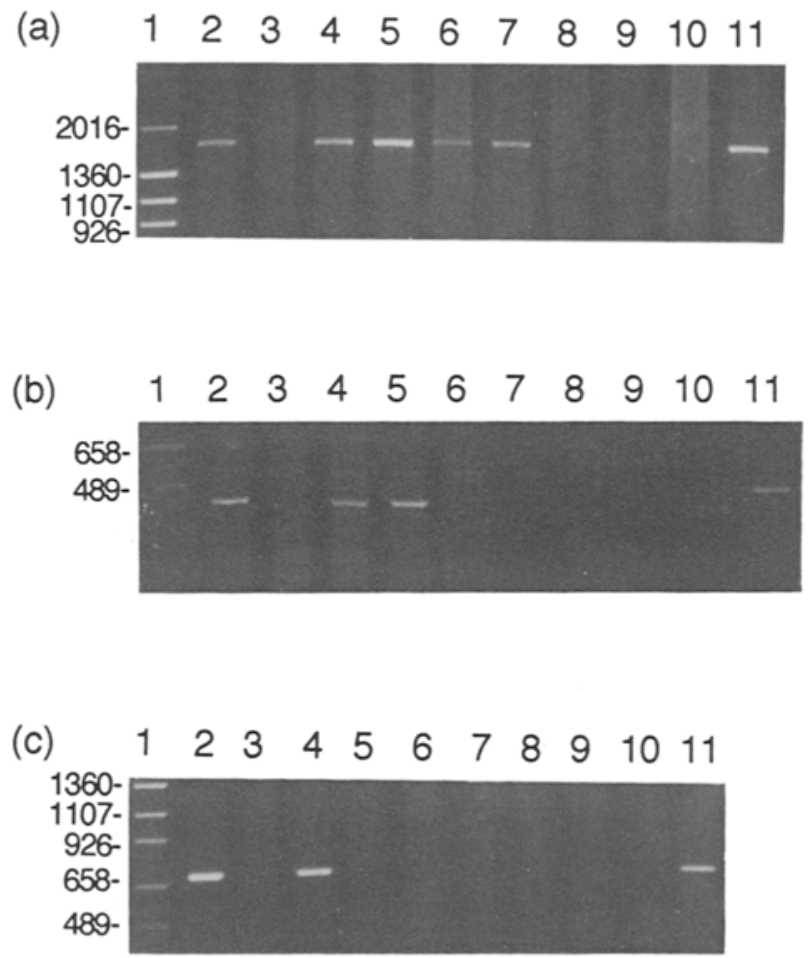

Fig. 1. Examples of the PCR products. Primer sets used were (a), DYS139; (b), DYS132, and (c), DYS1, respectively. In lanes 1 to 11 are pHY marker (Takara; Tokyo, Japan), male control, female control, and clinical samples 877, 1,027, $1,023,534,815,1,018,981$, and 924 . Size markers are indicated on the left in bp.

We describe here three sets of novel oligonucleotide primers which detect three loci, DYS139, DYS132, and DYS1, in intervals 5 to 6 of the Y chromosome. Very recently, a set of primers for DYS1 has been developed based on the 49f-related 49a sequence (Lucotte et al., 1991). An additional five sets of previously reported primers detect additional 5 loci (Ellis et al., 1990; Nakagome et al., 1991a, 1991b; Nakahori et al., 1991c; Witt and Erickson, 1989). The loci detected by these eight sets of primers are distributed over the nearly entire length of the Y chromosome. Results of detailed deletion mapping are also available (Nakahori et al., 1991a). There is an additional locus on Yp which is detectable by PCR (Tsukahara et al., 1990). Unfortunately, the locus has been vaguely mapped on Yp using only the in situ technique.

These eight sets of primers can be used for rapid detection of structually abnormal Y chromosomes in clinical samples. Such is crucial in the screening of patients who are at high risk for gonadoblastoma. This tumor develops in patients 


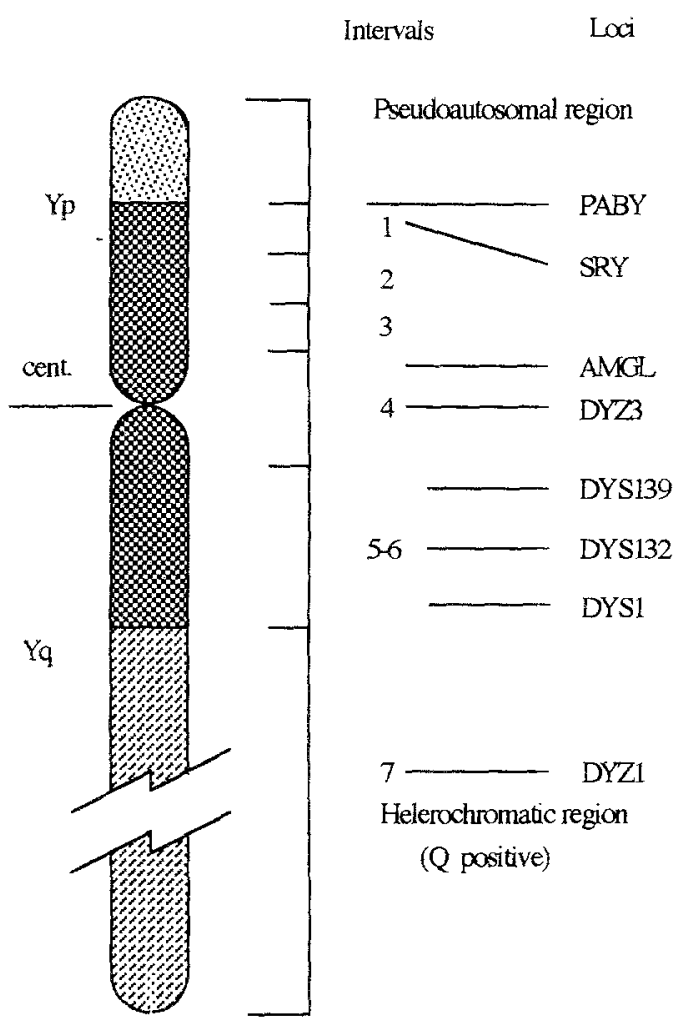

Fig. 2. The location and orders of the eight loci detected by PCR. Intervals 1-7 were defined by Vergnaud et al. (1986). The size of intervals are arbitrary.

with dysgenetic gonads who carry Y chromosome(s) or a part of it (Scully, 1970). Recently, we have shown that the development of gonadoblastoma is associated with a segment proximal to DYS132 (Nagafuchi et al., in press). When such patients have a marker chromosome, PCR analysis can quickly determine whether the marker is of $\mathrm{Y}$ origin, and therefore if the patient is at risk for gonadoblastoma.

Acknowledgments We are grateful to Dr. J. Weissenbach for probe $49 \mathrm{f}$ and to Dr. S.B. England for valuable discussion. This work is supported in part by grants from the Ministry of Health and Welfare, the Ministry of Education, Science and Culture, and Science and Technology Agency of Japan.

\section{REFERENCES}

Cooke H.I (1976): Repeated sequence specific to human males. Nature 262: 182-186

Ellis N, Taylor A, Bengtsson BO, Kidd J, Rogers J, Goodfellow P (1990): Population structure of the human pseudoautosomal boundary. Nature 344: 663-665

Human Gene Mapping 11 (1991): Cytogenet Cell Genet 58: nos. 1-4 
Lucotte G, David F, Mariotti M (1991): Nucleotide sequence of p49a, a genomic Y-specific probe with potential utilization in sex determination. Molec Cell Probes 5: 359-363

Nagafuchi S, Tamura T, Nakahori Y, Takano K, Nishi Y, Iwatani N, Kitao M, Hori Y, Konda S, Hasegawa T, Numabe H, Fujieda K, Tanaka T, Hibi I, Nakagome Y (1992): A majority of the marker chromosomes in Japanese patients with stigmata of Turner syndrome are derived from Y chromosomes. Human Genet 89: 590-592

Nakagome Y, Nagafuchi S, Seki S, Nakahori Y, Tamura T, Yamada M, Iwaya M (1991a): A repeating unit of the DYZ1 family on the human $\mathrm{Y}$ chromosome consists of segments with partial male-specificity. Cytogenet Cell Genet 56: 74-77

Nakagome Y, Seki S, Fukutani K, Nagafuchi S, Nakahori Y, Tamura T (1991b): PCR detection of distal Yp sequences in an XX true hermaphrodite. Am J Med Genet 41: 112-114

Nakahori Y, Mitani K, Yamada M, Nakagome Y (1986): A buman Y-chromosome specific repeated DNA family (OYZ1) consists of a tandem array of pentanucleotides. Nucleic Acids Res 14: 7569-7580

Nakahori Y, Tamura T, Nagafuchi S, Fujieda K, Minowada S, Fukutani K, Fuse H, Hayashi K, Kuroki Y, Fukushima Y, Agematsu K, Kuno T, Kaneko S, Yamada K, Kitagawa T, Nonomura M, Fukuda S, Kusano M, Onigata S, Hibi I, Nakagome Y (1991a): Molecular cloning and mapping of 10 new probes on the human $Y$ chromosome. Genomics 9: 765-769

Nakahori Y, Takenaka O, Nakagome Y (1991b): A human X-Y homologous region encodes "Amelogenin." Genomics 9: 264-269

Nakahori Y, Hamano K, Iwaya M, Nakagome Y (1991c): Sex identification by polymerase chain reaction using X-Y homologous primer. Am J Med Genet 39: 472-473

Saiki RK, Gelfand DH, Stoffel S, Scharf SJ, Higuchi R, Horn GT, Mullis KB, Erlich HA (1988): Primer-directed enzymatic amplification of DNA with a thermostable DNA polymerase. Science 239: 487--491

Scully RE (1970): Gonadoblastoma; A review of 74 cases. Cancer 25: 1340-1356

Sinclair AH, Berta P, Palmer MS, Hawkins JR, Griffiths BL, Smith MJ, Foster JW, Frischauf A, Lovell-Badge R, Goodfellow PN (1990): A gene from the human sex-determining region encodes a protein with homology to a conserved DNA-binding motif. Nature 346: 240-244

Tsukahara M, Matsuura S, Kishi F, Yoshida A, Kajii T (1990): Isolation of a Y chromosomal DNA sequence and its clinical application. Jpn J Hum an Genet 35 : 331-339

Vergnaud G, Page DC, Simmler M, Brown L, Rouyer F, Noel B, Botstein D, de la Chapelle A, Weissenbach $J$ (1986): A deletion map of the human $Y$ chromosome based on DNA hybridization. Am J Hum Genet 38: 109-124

Witt M, Erickson RP (1989): A rapid method for detection of Y-chromosomal DNA from dried blood specimens by the polymerase chain reaction. Hum Genet 82: 271-274 